Supporting Information for

\title{
Exciton Transfer and Emergent Excitonic States in Oppositely-Charged Conjugated Polyelectrolyte Complexes
}

William R. Hollingsworth, ${ }^{\neq}$Carmen Segura, ${ }^{\neq}$Jonathan Balderrama, Nathaniel Lopez, Pamela Schleissner, Alexander L. Ayzner*

University of California, Santa Cruz, Santa Cruz, CA

${ }^{*}$ Authors made equal contributions.

\section{S1. Photoluminescence Excitation of a Dilute CPEC Solution.}

Figure S1 show photoluminescence excitation (PLE) plots for a 1:0.25 charge ratio CPEC at a PFPI concentration of $0.1 \mathrm{mg} / \mathrm{mL}$. At this concentration, the PFPI exciton density as a function of position in the cuvette along the beam direction is significantly more uniform relative to the $1 \mathrm{mg} / \mathrm{mL}$ solution. This leads to much improved spatial overlap of emitted light with the cross section of the detector. Black diamonds show the PLE spectrum collected at $\lambda_{\mathrm{em}}=440 \mathrm{~nm}$, which corresponds to PFPI emission. The red curve shows PLE collected at $\lambda_{\mathrm{em}}=660 \mathrm{~nm}$, where PTAK exclusively emits. PFPI PL largely follows its OD (Figure 1 of main text). The fact that PTAK shows substantial enhancement in PL precisely at the wavelengths that give rise to PFPI PL constitutes strong evidence of EET from photoexcited to PFPI to PTAK. 


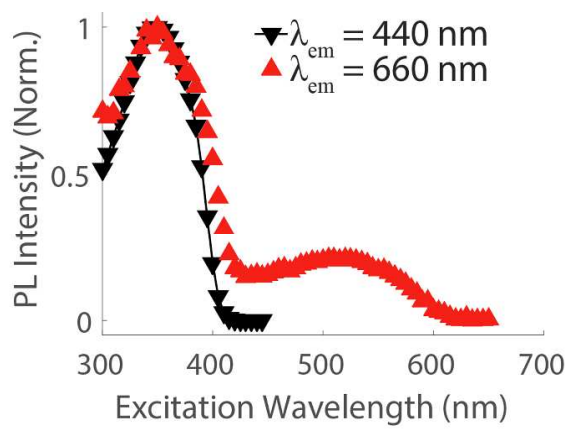

Figure S1. Normalized PLE of a 1:0.25 charge ratio CPEC. Black triangles show PL collected at $440 \mathrm{~nm}$, corresponding to PFPI PL. Red upsidedown triangles show PL collected at $660 \mathrm{~nm}$, corresponding to PTAK.

\section{S2. Fits to PL Spectra.}

In order to put a comparison of PL spectra between isolated PTAK control solutions and the corresponding CPEC solutions on a more quantitative footing, we have fit spectra on a photon energy scale to a vibronic progression. First, spectra were transformed from wavelength to energy space by scaling the measured intensity with a factor of $1 / E^{2}$, where $E$ is the photon energy. Intensities were further divided by a factor of $E^{3}$ to eliminate the energy dependence of the photon density of states. The resulting spectral intensities $I$ were fit to a sum of Gaussian functions to represent the different vibronic contributions to the PL envelope as

$$
\left.I=\sum_{n=0}^{3} a_{n} \exp \left[-\left(E-E_{0}+n E_{v i b}\right)^{2} / \sigma^{2}\right)\right]+ \text { const }
$$


where the $a$ 's are the Gaussian amplitudes, $E_{0}$ is the electronic origin, $E_{v i b}$ is the energy of the vibrational normal mode coupled to the electronic transition, and $\sigma$ is the width. A constant background offset was also included. The $0-0 / 0-1$ vibronic intensity ratio $\left(I_{0-0} / I_{0-1}\right)$ was calculated as the ratio of the first and second Gaussian amplitudes. The width was constrained to be constant for each vibronic peak but allowed to vary between samples. The fit results are summarized below in Table S1, along with the goodness-of-fit parameter $\mathrm{R}^{2}$.

Table S1. Gaussian fits to PL line shape in energy space.

\begin{tabular}{cccccc}
\hline Sample & $\mathbf{E}_{\boldsymbol{0}}(\mathbf{e V})$ & $\mathbf{E}_{\mathbf{v i b}}(\mathbf{e V})$ & $\boldsymbol{\sigma}(\mathbf{e V})$ & $\boldsymbol{I}_{\boldsymbol{0}-\boldsymbol{0}} / \boldsymbol{I}_{\boldsymbol{0}-\boldsymbol{1}}$ & $\mathbf{R}^{\mathbf{2}}$ \\
\hline PTAK $^{*}(1: 0.05)$ & 2.16 & 0.23 & 0.17 & 0.26 & 0.996 \\
CPEC $^{(1: 0.05)}$ & 2.01 & 0.13 & 0.10 & 0.91 & 0.999
\end{tabular}

* PTAK is the control for the corresponding CPEC (the charge ratio is indicated to the right).

It is at the moment unclear why the apparent energy of the vibrational mode coupled to PL transition of PTAK in isolation is substantially larger than that of PTAK complexed to PFPI. 